\title{
POLITIQUES PUBLIQUES ET ECONOMIE SOCIALE EN EUROPE LE CAS DE L'ESPAGNE
}

\author{
par \\ Rafael CHAVES* \\ Universidad de Valencia \\ CIRIEC-España
}

\begin{abstract}
RESUME**: Cet article examine les différentes politiques économiques susceptibles d'avoir des répercussions sur l'économie sociale en tant que secteur institutionnel. En premier lieu, il présente un cadre d'analyse permettant d'appréhender les contenus des politiques et en offre trois typologies. A la lumière de ce cadre, dans un premier temps il fait un bilan global des politiques mises en oeuvre en Europe, pour se centrer, dans un deuxième temps, sur l'Espagne, l'un des pays de l'Union où le niveau de développement de ces politiques est le plus avancé. Pour terminer, l'auteur évalue l'efficacité des principales mesures de politique économique mises en oeuvre dans ce pays.
\end{abstract}

\section{Introduction}

Envisagée globalement, l'économie sociale a une importante capacité à détecter et à satisfaire des besoins collectifs et à résoudre nombre de problèmes fondamentaux de nos sociétés. Ce potentiel se manifeste souvent dans des domaines socialement sensibles où tant le secteur public que le secteur privé capitaliste s'avèrent insuffisants ou inefficients. L'efficacité de l'économie sociale s'exprime principalement dans le domaine du développement et de l'autonomie des territoires et

\footnotetext{
* L'auteur remercie deux rapporteurs anonymes pour leurs remarques et commentaires.

** Abstract at the end of the article, Zusammenfassung am Ende des Artikels, resumen al fin del articulo.
} 
dans celui de la distribution équitable du revenu et de la richesse mais aussi par sa contribution à la résolution des problèmes d'emploi et par la production de services collectifs de bien-être social. Cette capacité à générer de la Valeur Ajoutée Sociale (Fraisse, Gardin, Laville, 2001) a été abondamment mise en avant par la littérature économique et sociologique (voir synthèse dans Sajardo, 1996a; Chaves, Monzón et Tomás-Carpi, 1999; Ruiz Olabuénaga, 2000; CIRIEC-International, 2000) et a principalement légitimé l'élaboration de politiques publiques en faveur des organisations et des entreprises de l'économie sociale.

Mais quelles sont les politiques qui peuvent être mises en place et quelles différences présentent-elles les unes par rapport aux autres? Quelles variables conditionnent leur mise en place effective? De quelle efficacité ont-elles fait preuve? Les études internationales qui ont abordé ces questions se sont multipliées ces dernières années suite à l'intérêt croissant pour l'économie sociale (ES) et ses deux grandes branches, les coopératives et les organisations à but non-lucratif. Cependant, il s'agit essentiellement d'études partielles. En effet, soit, elles n'abordaient que certaines branches de l'ES comme le secteur coopératif (Vienney, 1985; Rojo et Vidal, 1988; OIT, 2001) ou le secteur non-lucratif (Rose-Ackerman, 1986; James et Rose-Ackerman, 1986; Simon, 1990; Knapp, Robertson et Thomason, 1990; Sajardo, 1996), soit, elles se limitaient à étudier des politiques spécifiques comme celles des subventions et de la fiscalité (Clotfelter, 1985; Simon, 1987; Julià et Server, 1996), soit encore, elles se concentraient sur l'étude du processus d'élaboration de ces politiques (Dobkin, 1988; Kendall et Anheier, 1999; Fregidou-Malama, 2000).

Concernant l'ensemble de l'ES, de sérieuses carences se font sentir: manque d'analyses qui appréhendent globalement ces politiques, qui les évaluent, qui identifient les variables conditionnant leur mise en œuvre effective, et manque d'analyses portant sur l'Europe. De telles études sont particulièrement pertinentes aujourd'hui. En effet, l'ES a été confortée non seulement comme objet d'étude scientifique mais encore comme un grand acteur sur la scène sociale et économique européenne (CIRIEC-International, 2000). En outre, les différents décideurs politiques lui prêtent une attention croissante et lui accordent de plus en plus de place dans le cadre de la redéfinition des politiques publiques (Monnier et Thiry, 1997). Des études susceptibles de proposer des théories, des outils et des analyses consacrés à ces politiques présentent donc un grand intérêt. C'est dans cette perspective que se situe le présent travail.

À partir de deux récentes recherches collectives auxquelles l'auteur a pris part (CIRIEC-International, 2000; Barea et Monzón, 
2002), cet article envisage, sous différents angles, les politiques concernant l'ES. Nous proposons d'abord un cadre d'analyse qui permet d'appréhender les différentes politiques économiques (et, d'une manière générale, les politiques publiques) susceptibles d'avoir des répercussions sur l'ES en tant que secteur institutionnel. A la lumière de ce cadre d'analyse, nous présentons ensuite une synthèse des principaux résultats des deux recherches citées, en commençant par un bilan des politiques mises en œuvre en Europe. Ensuite, l'analyse se concentre sur l'Espagne, l'un des pays de l'Union où le niveau de développement de ces politiques est le plus avancé, en ciblant l'économie sociale marchande. Nous examinons les politiques adoptées dans ce pays méditerranéen, nous évaluons l'efficacité des principales mesures de nature strictement économique et nous analysons le rapport entre le niveau d'effort budgétaire de soutien de l'économie sociale et les indicateurs politique et de développement économique des régions.

\section{Typologies des politiques concernant l'économie sociale}

L'espace occupé par l'économie sociale dans le système économique, c'est-à-dire son espace systémique, dépend directement de la vision et de l'attitude que les décideurs politiques ont envers le secteur ainsi que de celles des autres acteurs sociaux participant au processus d'élaboration et d'application des politiques publiques. Il dépend aussi du rôle qui est octroyé à l'économie sociale dans le système, ainsi que des politiques de soutien qui lui sont destinées. En d'autres mots, cet espace est conditionné (mais non déterminé) par les politiques publiques mises en ouvre dans le secteur de l'économie sociale.

Selon la théorie de la politique économique, toute politique est basée sur un corps théorique et des valeurs et est conditionnée par une structure socio-économique et des institutions déterminées. Les valeurs et le cadre théorique de référence définissent les objectifs publics généraux à atteindre par les politiques ainsi que les instruments a priori susceptibles d'être utilisés. La structure et les institutions définissent par contre la réalité spécifique dans laquelle doivent se déployer les politiques, réalité qui à son tour définit les problèmes socio-économiques à résoudre, les acteurs du processus politico-économique et leurs rapports de pouvoir, les règles du jeu, le cadre culturel et idéologique, et enfin, la priorité à donner aux politiques (objectifs et instruments) effectivement envisageables (Tomás-Carpi, 1990; Chaves et Monzón, 2000a). 
Cette section a pour objectif de dresser une grille d'analyse des contenus des politiques à l'égard de l'ES. Trois approches sont retenues. ${ }^{1}$ La première distingue les aspects liés à l'offre et à la demande de l'action économique publique. La deuxième distingue le caractère transversal du caractère sectoriel des politiques déployées. Finalement, la troisième approche classe l'ensemble des politiques déployées en fonction de leur orientation globale.

C'est la dimension du secteur institutionnel qui est l'axe de référence. L'action économique publique visant un secteur institutionnel particulier, comme celui de l'ES, présente des particularités nécessitant des politiques distinctes de la politique économique générale, qui porte sur l'ensemble de l'économie, et des politiques sectorielles qui visent les différents secteurs d'activité économique (agriculture, industrie, services ...). En effet, un secteur institutionnel se définit par les formes juridiques et organisationnelles qui le composent ${ }^{2}$ et constitue une partie seulement du système économique. ${ }^{3}$ Il déborde généralement du champ d'un secteur d'activité économique, raison pour laquelle les politiques sectorielles (la politique agricole, par ex.) ne l'affectent que partiellement. Chaque secteur institutionnel, à savoir, l'économie sociale, le secteur public et le secteur privé capitaliste (voir Defourny et Monzón, 1992) est en concurrence avec les autres secteurs et développe des alliances afin d'accroître son espace systémique. ${ }^{4}$

2.1 Politiques d'offre et politiques de demande concernant l'économie sociale

Comme pour la politique économique générale, deux types de politiques peuvent être distingués, les politiques d'offre et les politiques de demande (cf. Tableau 1).

1 Cette analyse s'inspire largement de CIRIEC-International, 2000, Chap. 4. 2 Bien que des formes mixtes entre les trois secteurs institutionnels puissent être créées (voir Monnier et Thiry, 1997).

3 En réalité un secteur institutionnel, en particulier celui de l'ES, n'est pas uniforme du point de vue institutionnel mais significativement hétérogène dans sa composition. Chaque famille interne ou branche (par ex. les coopératives) constitue un véritable sous-secteur institutionnel qui justifie qu'on lui attribue un traitement politique propre.

4 En ce sens, des gains d'espace systémique de la part du secteur privé capitaliste signaleraient un processus de privatisation tandis que des gains de la part du secteur public signaleraient un processus de nationalisation. 


\section{Tableau 1 - Politiques d'offre et politiques de demande concernant l'économie sociale}

\begin{tabular}{ll} 
Politiques d' offre visant la STRUCTURE des Organisations de l'économie sociale \\
\hline $\begin{array}{l}\text { Politiques } \\
\text { institutionnelles }\end{array}$ & Reconnaissance en tant qu'acteur privé \\
& - Reconnaissance de la liberté d'action dans tous les secteurs \\
& économiques \\
- & Elimination/compensation des entraves juridiques due à l' usage de \\
& statuts propres au secteur (par ex. par des mesures d'ordre fiscal) \\
- & Reconnaissance en tant qu'acteur du processus d'élaboration des \\
& politiques publiques \\
- & Reconnaissance en tant qu'acteur du processus d'application des \\
& politiques publiques \\
- & Appui à la reconnaissance médiatique du secteur par le grand public \\
- Enseignement/Recherche: Insertion dans le système d'éducation et & dans les programmes de recherche financés par les fonds publics. \\
Politiques d'ordre & - Modes de régulation visant à réduire le co't des facteurs productifs \\
cognitif & (capital et travail) et à améliorer l'accès au financement extérieur \\
Politiques & privé \\
financières & Budgets publics de soutien à l'économie sociale \\
& Existence de structures publiques de soutien à l'économie sociale qui \\
offrent des services réels : information, formation, Networking, recherche, \\
conseil technique, commercial, ...
\end{tabular}

Politiques de demande: visant l'ACTIVITÉ des Organisations de l'économie sociale

Mesures qui favorisent l'économie sociale en tant que fournisseur des Administrations

Publiques.

- Meilleur accès au statut de fournisseur (par ex. garanties moins importantes pour accéder aux appels d' offre publics, clause de préférence - en cas d'égalité - dans les appels d' offre, pratiques de 'discrimination' positive de l'ES dans les appels d' offre sur base de clauses sociales ou d' insertion)

- Modes de régulation et de mise en oeuvre de partenariats entre ES et pouvoirs publics qui ne lèsent pas la première (par ex. durabilité des dispositifs, définition à long terme des budgets affectés, politique de paiement plus favorable - avances, courts délais de paiement, etc.)

Les politiques d'offre visent les structures des organisations de l'ES. Elles comprennent les politiques institutionnelles et les politiques d'ordre cognitif. Ces dernières visent à lui conférer une plus grande visibilité et réceptivité sur le plan culturel. Les politiques institutionnelles visent à attribuer un espace systémique au secteur à partir du cadre institutionnel. Les mesures de ce type tendent à l'institutionnalisation de certaines formes organisationnelles spécifiques et à leur non discrimination par rapport aux autres formes juridiques. Cette approche transversale et globale implique 1) la reconnaissance de l'identité différenciée de ces formes 
organisationnelles (par ex. les lois sur les coopératives, les mutuelles et les associations), 2) la reconnaissance de leur capacité d'action dans tout secteur d'activité économique, 3) l'élimination ou la compensation des difficultés à opérer sous ce statut particulier qui les désavantage (position qui peut être corrigée par une fiscalité favorable, par exemple,$^{5}$ cf. Julià et Server, 1996), et enfin 4) leur reconnaissance en tant qu'interlocuteur dans le processus d'élaboration et d'application des différentes politiques.

Par ailleurs, les politiques d'offre comprennent les politiques visant à promouvoir l'efficience et l'efficacité de l'ES au moyen de supports matériels, articulés autour de dispositifs financiers et de structures d'appui technique. Dans cette approche, les administrations publiques concernées par le développement des coopératives et de l'ES ont deux options : soit, établir un environnement régulateur, cognitif et financier, favorable à l'éclosion et au développement d'initiatives dans le secteur (environmental approach, dans la littérature anglo-saxonne, cf. Chaves, 1999), soit, au-delà de la création de ce contexte, adopter un rôle proactif en intervenant dans la promotion directe du secteur (entrepreneurial approach). Cette proactivité de la politique économique apparait surtout lorsque l'initiative privée est particulièrement faible et souffre de carences fonctionnelles ou dans le but de raccourcir les périodes de maturation des processus économiques, ce qui peut être particulièrement opportun dans des contextes de transformation économique accélérée comme celui que nous connaissons. Dans le cadre de cette seconde option, les pouvoirs publics mobilisent des ressources matérielles et humaines dans le but d'offrir au secteur de l'ES un éventail de services réels (non financiers), s'adaptant aux besoins (services en matière d'information, de formation, de recherche, de conseil, de networking, de restructuration et de soutien à la création de structures de 2ème degré, etc).

La politique de demande, de conception keynésienne, porte quant à elle sur les activités économiques déployées par les organisations de l'ES. Elle part du principe qu'une augmentation des dépenses achats publics aux organisations de l'ES - accroît leur niveau d'activité. Cette politique vise à réguler et stabiliser le secteur en tant que fournisseur du secteur public. En effet, les administrations

$5 \quad$ La fiscalité favorable à certaines formes de l'ES peut dès lors se justifier comme un mécanisme de compensation et non de promotion. 
publiques sont le principal client des économies développées; les achats publics (marchés publics) représentent environ 10\% du PIB des pays européens (AAVV, 2000). A l'époque du fordisme, les achats publics étaient l'un des outils des politiques de demande qui visaient à réguler le niveau d'activité. La désuétude dans laquelle sont tombées ces politiques est intimement liée aux effets de débordement croissants, propres aux économies ouvertes. Ce type de politiques reste néanmoins utile au niveau des secteurs institutionnels. Si elles sont mises en place en sélectionnant des activités économiques à faible effet de débordement et des secteurs institutionnels plus enracinés sur le territoire, elles conservent toute leur pertinence. Sous cet angle, l'ES s'en trouve de toute évidence revalorisée.

Cette politique de demande repose aussi sur un autre argument. Dans un contexte de libre marché et de libre concurrence, l'égalité des chances d'accès aux marchés publics est le minimum que les administrations publiques doivent garantir aux différents opérateurs, conception par ailleurs soutenue par l'Union Européenne. En l'absence de disposition légale, cette condition n'est pas remplie car les grandes entreprises privées traditionnelles jouissent d'avantages d'accès importants par rapport aux entreprises de l'ES. Cette situation peut être corrigée en améliorant leur accessibilité par des mesures réduisant les coûts des garanties nécessaires, en adaptant le mode de paiement ou encore en incluant certaines clauses spécifiques dans les contrats. Sur ce dernier point, l'administration peut jouer un rôle crucial. En effet, si l'on part du principe que les administrations publiques doivent intervenir comme client consommateur responsable guidé par l'intérêt général - le choix des fournisseurs ne peut rester neutre. Par conséquent, dans le respect des exigences de qualité et de prix, elles doivent privilégier les formes d'organisations les plus aptes à satisfaire l'intérêt social et à créer de la valeur ajoutée sociale (emploi, équité dans la distribution, cohésion sociale...).

2.2 Politiques transversales et politiques sectorielles concernant l'économie sociale

Nous basant sur la dimension de secteur institutionnel, les politiques transversales présentent comme champ d'action l'ensemble d'un secteur institutionnel, celui de l'ES dans le cas présent. Elles visent à faciliter la présence et la capacité d'action de l'ensemble des entreprises du secteur dans le système. 
À la différence des politiques transversales, les politiques sectorielles $^{6}$ portent sur un aspect restreint de la réalité socioéconomique, aspect défini par la nature des nécessités sociales et des problèmes importants auxquels la politique économique tente de répondre. On parle de la politique agricole, de la politique de l'emploi, de la politique sociale, de la politique en matière d'éducation, etc. Elles sont conçues généralement par un seul ministère - département. Une partie seulement du secteur de l'ES, les organisations attachées à la problématique du secteur d'activité économique, s'insère dans les politiques sectorielles (par ex. les coopératives agricoles dans le cadre de la politique agricole, les ONG de développement dans le cadre de la politique sociale de coopération au développement, ...).

2.3 Politiques d'ordonnancement et politiques de processus à l'égard de l'économie sociale

Une troisième typologie des politiques publiques, héritière de l'école allemande, ${ }^{7}$ repose sur l'orientation globale de l'ensemble des politiques. Elle distingue les politiques d'ordonnancement (Ordnungspolitik) des politiques de processus (Prozesspolitik).

Les premières, conceptuellement proches des politiques de réformes structurelles de Tinbergen, visent à établir ou modifier le 'cadre' ('ordnungs') du système économique. Elles affectent les institutions et les règles centrales de fonctionnement du système. Marquées par les valeurs fondamentales et les objectifs généraux de long terme d'une société, elles définissent les caractéristiques centrales du système, comme les niveaux de centralisation ou décentralisation du secteur public, l'espace réservé au marché et à la planification publique, les agents économiques et les règles du jeu. Les politiques de processus visent à résoudre les problèmes et déséquilibres engendrés par le fonctionnement du système (le processus économique ou 'prozess'). Elles fonctionnement logiquement encadrées par les politiques d'ordonnancement.

6 Dans la seconde partie de l'article (sections 3 et 4) seule l'institulionnalisation de la politique sectorielle de l'emploi a été analysée. Au niveau européen, cela s'explique par l'objet de l'étude qui était la contribution à l'emploi de l'ES. Pour l'Espagne, c'est parce que les décideurs politiques consultés étaient les responsables en matière d'emploi.

7 Eucken, Ropke et Muller-Armack (voir Jané Solà, 1979; Vanberg, 1988; Cox, 1997; Cuadrado, 2001) furent des représentants de ce courant. 
Cette troisième typologie des politiques revalorise la dimension 'secteur institutionnel' qui est étroitement liée au cadre économique et institutionnel. L'importance de ce cadre est double. D'une part, parce qu'il définit les marges de manoeuvre de la prozesspolitik. D'autre part, parce qu'il définit les problèmes économiques propres à chaque système auxquels devra faire face la politique économique. De plus, situer le débat de la politique économique dans le champ de la ordnungspolitik exige d'introduire le concept d'efficience socioéconomique. Le choix du cadre ne répondra pas exclusivement à des objectifs économiques (efficience économique) mais aussi à des finalités socio-politiques (efficience socio-politique) (Cox, 1997; Tomás-Carpi, 1997). Différents types de cadres devront être analysés par rapport à des valeurs ou groupes de valeurs (par ex. la liberté positive, la démocratie, l'égalité des chances, etc.). Situer le débat sur ce plan revalorise significativement le rôle de l'ES dans le système.

Les politiques d'ordonnancement à l'égard de l'ES se déploient quand il existe une ample reconnaissance des atouts du secteur en tant que vecteur d'action positive sur le bien-être social et en tant que porteur de culture et de valeurs socialement souhaitables. Quand une conception de ce genre prévaut parmi les protagonistes du processus politique, les politiques publiques sont des politiques de long terme menées pour instaurer un cadre structurel institutionnel, culturel et matériel adapté pour le développement de ce secteur. S'instaurent alors des mesures stables et structurelles du système socioéconomique et non des mesures sujettes aux cycles politiques et économiques. Dans les pays européens, ces politiques se déploient rarement dans une perspective transversale mais plutôt dans une perspective sectorielle.

Les politiques de processus se développent, par contre, quand on reconnaît au secteur un nombre réduit d'atouts ou de contributions à l'intérêt général. Seules certaines propriétés fonctionnelles pour la poursuite d'objectifs spécifiques de politique publique sont reconnues. C'est le cas de la reconnaissance de l'utilité des coopératives de travail comme instrument dans la politique de l'emploi, tout en niant ses autres propriétés. Les politiques de processus d'appui au secteur de l'ES se traduisent généralement par des mesures de promotion matérielle économico-financière. Elles sont pour la plupart transitoires et s'insèrent dans les politiques sectorielles en demeurant actives uniquement durant la résolution d'un problème déterminé (par ex. le chômage). 
Tableau 2 - Politiques d'ordonnancement et politiques de processus à l'égard de l'économie sociale

\begin{tabular}{|c|c|c|}
\hline Vision de l'économie sociale & & Types de Politiques \\
\hline $\begin{array}{l}\text { Economie sociale } \\
\text { considérée comme } \\
\text { OBJECTIF: } \\
\text { (souhaitable en elle- même; } \\
\text { reconnaissance large de se } \\
\text { atouts) }\end{array}$ & $\begin{array}{l}\text { Politiques } \\
\text { d'Ordonnancement }\end{array}$ & $\begin{array}{l}\text { - Les mesures visant l'économie sociale se } \\
\text { situent dans le contexte institutionnel et } \\
\text { cognitif du pays: } \\
\checkmark \text { mesures de reconnaissance et d'appui } \\
\text { légal (par ex. statuts, fiscalité, régulation de } \\
\text { l'accès aux marchés publics) } \\
\checkmark \text { mesures d' appui matériel (aides } \\
\text { financières, services réels fournis par l'Etat) } \\
\checkmark \text { mesures d'ordre cognitif (diffusion } \\
\text { médiatique, introduction dans les } \\
\text { programmes éducatifs et de recherche) }\end{array}$ \\
\hline $\begin{array}{l}\text { Economie sociale } \\
\text { considérée comme } \\
\text { INSTRUMENT: } \\
\text { (souhaitable en tant } \\
\text { qu'instrument utile pour } \\
\text { d'autres objectifs; } \\
\text { reconnaissance étroite de } \\
\text { ses atouts) }\end{array}$ & $\begin{array}{l}\text { Politiques de } \\
\text { Processus }\end{array}$ & $\begin{array}{l}\text { - Les mesures visant l'économie sociale } \\
\text { sont subordonnées à d' autres politiques } \\
\text { publiques } \\
\text { (par ex. les mesures d'appui aux: } \\
\text { - Coopératives de travail dans le contexte } \\
\text { des politiques de l'emploi; } \\
\text { - Associations dans le contexte des } \\
\text { politiques sociales; } \\
\text { - Coopératives agricoles dans le contexte } \\
\text { des politiques agricoles.) }\end{array}$ \\
\hline
\end{tabular}

Source: Chaves, Monzón et Tomás-Carpi (1999).

\section{$3 \quad$ Les politiques mises en œuvre en Europe}

L'étude des politiques publiques à l'égard de l'ES déployées en Europe était un des trois buts principaux de la récente recherche internationale (CIRIEC-International, 2000). Les principaux résultats issus de cette étude sont synthétisés et analysés dans la présente section.

Afin de classer les différents pays européens en fonction du type de politiques $^{8}$ visant l'ES ainsi qu'en fonction du degré de déploiement de celles-ci, nous avons utilisé la méthode suivante. Nous avons considéré

8 L'information disponible était insuffisante pour trois pays (l'Autriche, la Finlande et le Luxembourg), raison pour laquelle ils ont été exclus de cette étude comparative. 
les politiques exposées dans le Tableau 1, à l'exception des politiques d'ordre cognitif qui ne sont pas strictement économiques et qui n'ont pas fait pas l'objet de l'étude transnationale. Les mesures de politique de demande considérées au Tableau 3 sont celles qui facilitent explicitement l'accès de l'ES aux marchés publics. On a inclu également l'analyse de la politique sectorielle de l'emploi, considérant ici l'existence de mesures visant à soutenir l'emploi dans l'ES en intégrant explicitement ses branches dans la politique nationale de l'emploi. Pour pondérer le niveau de déploiement des différentes politiques nous suivons la méthodologie proposée par Thiry et Vivet (2000) qui utilisent les symboles $0,+,++,+++$; trois + ne représentent pas un maximum d'appui absolu mais signalent un maximum relatif en Europe. Le symbole (C) indique que l'appui au mouvement coopératif est reconnu dans la Constitution Nationale. On a différencié le traitement des deux branches de l'ES, les Coopératives-Mutuelles (SCoop) et le Secteur à but non lucratif (SNL). Enfin, afin de mettre en relation le degré de déploiement des politiques avec d'autres variables pertinentes des pays, on a inclu dans le Tableau 3 le niveau de l'emploi par branches de l'ES et par pays ainsi que le degré de reconnaissance du secteur par les pouvoirs publics.

A la lecture du Tableau 3, on constate que ces politiques se déploient de façon inégale dans les pays de l'Union et selon les branches et présentent des contenus fort différents. En effet, on observe que le développement des politiques publiques à l'égard de l'ES est plus avancé dans les pays méditerranéens latins (France, Italie, Espagne et Portugal) que dans les pays du nord de l'Europe. L'ampleur et l'importance des politiques effectivement mises en place sont étroitement liées à la reconnaissance sociale et politique de l'ES, à la visibilité et à l'image du secteur dans la société et auprès de décideurs politiques, au rôle qu'il joue dans le développement multidimensionnel (économique, social, culturel) des pays, à son poids économique et à la tradition de cette réalité, et finalement à sa capacité à être un interlocuteur valable dans les différents processus d'élaboration et d'application des politiques publiques.

Dans le premier groupe de pays, qui forme le modèle méditerranéen latin (à l'exclusion donc de la Grèce), les politiques institutionnelles déployées sont très développées. Dans trois pays, cette institutionalisation s'inscrit même dans la Constitution Nationale. Il existe un biais général favorable aux coopératives, depuis la fiscalité jusqu'à l'insertion du secteur dans la politique sectorielle de l'emploi, ce qui se traduit par un poids relatif plus important de l'emploi coopératif par rapport au total de l'emploi du secteur. 


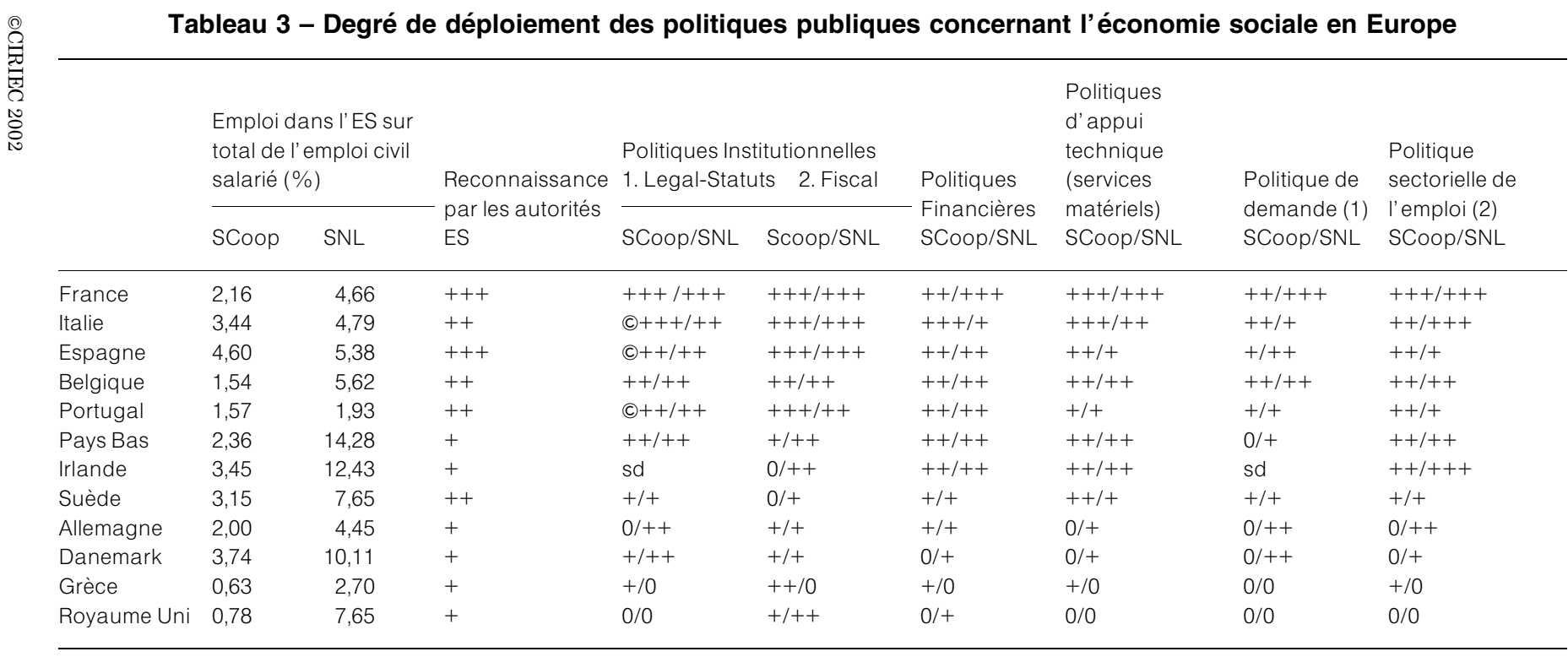

Source: CIRIEC-International. Année de référence du taux d'emploi de l'ES: 1995.

Notes: (1) Soutien explicite à l'accès des organisations de l'ES aux marchés publics; (2) Soutien explicite dans les politiques de l'emploi. 
Le modèle germano-britannique de développement des politiques, dont l'Allemagne, le Royaume Uni, l'Irlande et le Danemark sont les pays représentatifs, se caractérise par contre par un déploiement relativement moins important de mesures de soutien, et dans ce cadre, par un traitement plus favorable aux organisations à but non lucratif qu'aux coopératives. En outre, il existe une tendance à la réduction du soutien aux coopératives - ainsi la réduction de l'appui fiscal aux coopératives allemandes - comparativement à la situation dans les pays latins. Par ex. au Portugal et en Italie le soutien aux coopératives a augmenté significativement ces dernières années. Dans les pays issus du modèle germano-britannique, la prépondérance de l'emploi relatif des SNL est plus marquée.

La Suède, la Belgique et les Pays Bas se situent dans une position intermédiaire entre les deux modèles précédents. Enfin, la Grèce conjugue un très faible développement de politiques concernant l'ES (avec un traitement relativement plus favorable pour les coopératives) à un taux d'emploi dans l'ES par rapport au total de l'emploi civil salarié le plus faible de l'Europe.

Les politiques d'offre sont plus développées que celles de demande en Europe, bien que l'on assiste à une expansion de ces dernières. La politique de demande est principalement opérationnelle dans les services sociaux, grâce aux contrats de courte durée passés avec le secteur de l'ES, notamment avec les organisations sans but lucratif, et grâce à des subventions conditionnées au développement de ces activités. Par contre, la politique de demande a de sérieuses difficultés à s'institutionnaliser, par exemple, par l'inclusion de clauses sociales dans les contrats publics.

La France et l'Italie présentent le modèle de politiques publiques le plus avancé de l'Europe en matière de soutien à l'ES. La politique d'ordonnancement des deux pays révèle un large cadre institutionnel et culturel d'appui au secteur, aussi bien du point de vue des politiques d'offre et de demande que de la politique de l'emploi, bien que l'Italie présente un biais favorable aux coopératives. Dans les autres pays correspondant au modèle méditerranéen latin, on observe un sérieux effort pour élaborer des politiques d'ordonnancement plus solides. Ce panorama s'oppose à celui du reste des pays de l'Europe du Nord, où les politiques déployées sont des politiques de processus, peu vigoureuses et très sujettes aux priorités politiques de court et moyen termes. 


\section{Les politiques concernant l'économie sociale marchande en Espagne}

L'emploi de l'ES marchande ${ }^{9}$ représentait, en Espagne, 2,42\% du total de la population occupée en 2000 (PO). On observe au Tableau 5 que le chiffre le plus élevé de ce "taux de coopérativisation" était détenu par le Pays Basque, fief du célèbre groupe Mondragón Corporación Cooperativa - MCC - où il atteignait 7,24\%, soit 58.116 emplois. ${ }^{10}$ Le Pays Basque est suivi par la Navarre et la Murcie.

L'évolution au cours de la période 1997-2000 a été une croissance nette ce qui, dans un contexte économique d'expansion, indique que l'ES a cru plus que ne l'a fait le reste de l'économie. Toutes les régions ont gagné de l'espace systémique (cf. Tableau 5), ${ }^{11}$ mais la croissance la plus élevée pour la même période est, à nouveau, celle du Pays Basque, suivi par la Murcie et par la Navarre. Le Pays Basque a gagné 1,65\% d'espace systémique, grâce à nouveau au fort dynamisme du groupe coopératif MCC.

Si le dynamisme même du secteur de l'ES et de la société civile ont été, sans aucun doute, des facteurs déterminants de cette expansion, le rôle des politiques mises en place n'a pas été moins important. Ces politiques sont analysées ${ }^{12}$ dans cette partie de l'article qui s'inspire du Rapport sur la situation de l'ES en Espagne en 2000 (cf. synthèse dans Barea et Monzón, 2002). Le mode de déploiement des politiques publiques visant l'ES en Espagne répond bien au modèle méditerranéen latin exposé dans la section précédente. Certaines

9 Dans cette section l'analyse des politiques publiques est approfondie en en limitant le champ, géographiquement, en portant sur l'Espagne, et par branche institutionnelle, en visant l'ES marchande. L'ES marchande inclut en Espagne les coopératives et les sociétés de travailleurs (sociedades laborales SLab) mais exclut les mutuelles qui y sont peu présentes. Cette définition du champ coïncide avec la conception dominante de la réglementation en vigueur à ce jour dans les Administrations publiques et avec la conception des décideurs politiques responsables en matière de soutien de l'ES (cf. Questionnaire-CIRIEC).

10 La variable EES du Tableau 5 représente l'emploi de l'ES en l'an 2000.

$11 \Delta$ EES, dans le Tableau 5, représente l'emploi de l'ES en 2000 moins l'emploi de l'ES en 1997.

12 La période de référence est celle de la fin des années 90 et plus particulièrement l'année 2000. Par ailleurs, et comme pour l'Europe, les politiques d'ordre cognitif n'ont pas été prises en compte. Pour l'élaboration du chapitre sur les politiques publiques du rapport Barea et Monzón (2000), outre l'information puisée dans les publications officielles et dans les documents 
politiques institutionnelles sont très solides et des efforts sont faits pour développer les politiques d'ordonnancement.

\subsection{Politiques institutionnelles}

En Espagne, grâce à l'Article 129.213 de la Constitution de 1978, et à un modèle composé de plusieurs gouvernements compétents en la matière, les mesures institutionnelles déployées envers l'ES ont été multiples.

En ce qui concerne la reconnaissance de l'identité différenciée de ces organisations, on peut parler d'une véritable inflation législative. En effet, un nombre croissant de régions - onze à l'heure actuelle, nommées Communautés Autonomes (CCAA) - ont progressivement réglementé le statut des coopératives et multiplié les textes législatifs. Des problèmes de concurrence entre les administrations centrale et autonomes (dans le cas des SLab) ont mis un frein à cette inflation dans le secteur.

La nouvelle réglementation régionale et étatique des coopératives et des SLab a favorisé la multiplication de ce type de structures. Un facteur particulièrement propice a été la réduction du nombre minimum des membres nécessaires à la constitution des SLab et des coopératives ouvrières de production (CTA) ainsi que la réduction du capital minimum (de 600.000 à 3.000 Euros) pour la création d'une SLab. En effet, si en 1996, le nombre des SLab créées en Espagne n'était que de 706, en 2000 il s'élève à 4851. En 1997, la législation sur les SLab a été modifiée. D'un autre côté, la Catalogne est la première région à avoir en 1991 réduit à 3 le nombre minimum de membres nécessaires à la constitution d'une CTA. En 1991, 136 CTA sont créées

internes des Administrations, le CIRIEC-Espagne a recueilli une information directe par le biais de deux questionnaires auprès des responsables politiques des Administrations autonomes régionales compétents en matière de développement de l'ES. Le premier consistait en un questionnaire ouvert demandant aux responsables interrogés d'évaluer leur propre politique récente en la matière et le second, que nous avons appelé Questionnaire-CIRIEC, semifermé, présentait à toutes les Communautés Autonomes (dorénavant CCAA) une liste exhaustive de questions homogènes dont les réponses étaient, en revanche, libres.

13 'Les pouvoirs publics promouvront efficacement les différentes formes de participation dans l'entreprise et favoriseront par le biais d'une législation adéquate, les sociétés coopératives. Ils adopteront aussi les dispositifs facilitant l'accès des travailleurs à la propriété des moyens de production', Art. 129.2 Constitution Espagnole, Royaume d'Espagne. 
dans cette région, soit $14 \%$ du total du pays et 855 en 1993 , soit $40 \%$ du chiffre national, taux inchangé jusqu'à ce jour.

Certaines résistances s'opposent néanmoins à l'apparition de nouvelles formes de structures d'ES, comme les entreprises d'insertion ou les coopératives sociales et d'utilité publique au sens large (à l'instar de l'Italie).

Pour ce qui est de la capacité théorique d'agir dans les différents secteurs d'activité économique, des obstacles s'élèvent pour les coopératives, en particulier (cf. Noticias del CIDEC no. 33) dans les secteurs électrique et de distribution d'hydrocarbures.

Les différentes formes d'ES jouissent d'un contexte fiscal très favorable bien que variable en fonction des formes juridiques (pour une comparaison avec les autres pays européens, voir Sajardo, 1996b; Commission, 1997 et DIES, 2001). La loi principale en la matière, la Loi 20/1990 sur la fiscalité des coopératives accorde des avantages fiscaux importants; elle n'a cependant pas été conçue comme un mécanisme de compensation mais comme un moyen de promotion. Il convient de signaler une certaine incohérence au sein de la réglementation. Ainsi, des formes hybrides entre les coopératives et les institutions à but non lucratif, d'utilité sociale ou d'intérêt public (par ex., les coopératives non lucratives), qui font l'objet de huit lois.

Les carences les plus lourdes se manifestent dans l'institutionnalisation de l'ES comme interlocuteur de la concertation sociale pour l'élaboration et l'application des politiques. Sa présence reste marginale tant au sein des Conseils économiques et sociaux national et régionaux, que dans les Pactes régionaux pour l'emploi et le développement économique (Aragon, 2000). La défense des intérêts de l'ES se fait souvent par l'intermédiaire de l'un des grands interlocuteurs sociaux, les syndicats. Cette situation réduit les opportunités de l'ES de pouvoir jouer un rôle dans d'importantes sphères de la vie socioéconomique.

D'autres mécanismes ont été quant à eux institutionnalisés. Ainsi, les organes de concertation, tant au niveau national (c'est le cas du Conseil de Développement de l'ES) qu'au niveau régional (avec les Conseils supérieurs des coopératives) sont mentionnés dans les lois des Communautés Autonomes sur les coopératives. Mais leur jeune âge, pour la plupart, rend une éventuelle évaluation encore prématurée.

\subsection{Politiques financières}

L'économie sociale présente des problèmes structurels d'ordre financier qui s'expliquent, en partie, par les règles statutaires 
spécifiques de fonctionnement interne (système de partage du pouvoir de décision et principe de distribution des bénéfices qui tendent à décourager les investisseurs privés) et, par les difficultés à accéder aux marchés financiers privés. Ce facteur d'étranglement peut être atténué - voire même neutralisé - par des mesures financières publiques.

Un premier groupe de mesures financières a été adopté par le biais de la nouvelle législation sur les coopératives. Leur but est de renforcer les fonds propres des coopératives en ouvrant le capital social aux investisseurs extérieurs et en rendant plus flexibles les règles internes, par des formules comme celle des membres collaborateurs et partenaires. En outre, ces mesures permettent l'émission par les coopératives, auprès du grand public, de titres d'emprunts sans droit de vote. Les répercussions de ces mesures sont, à ce jour, encore très limitées.

Une autre mesure financière consiste à favoriser la construction de structures de soutien financier liées au secteur de l'ES. Un exemple traditionnel est celui des sections de crédit internes aux coopératives agricoles, dont le but est de renforcer leur position financière. La législation récente de la Banque d'Espagne et de quelques gouvernements autonomes, comme celui de Valence, a cependant nuit à l'activité de ces sections et a même provoqué leur disparition progressive en exigeant d'elles d'élargir l'éventail des destinataires des prêts à des personnes étrangères aux coopératives. Le développement du financement alternatif (cf. Noticias CIDEC no. 33) lié au financement éthique et au microcrédit, est une nouvelle formule de soutien financier à l'ES, mais qui n'a, encore, qu'un faible écho.

A l'instar d'autres pays européens comme l'Italie, certaines lois coopératives comme les lois catalane et galicienne, introduisent un autre type de financement qui implique les Caisses d'épargne. Ces dernières, en tant que fondations financières à intérêt social, doivent destiner une partie de leurs investissements au secteur coopératif.

Les mesures financières peuvent aussi se situer dans le domaine de la réglementation du travail. Ainsi, l'assouplissement ou l'annulation, pour des travailleurs de l'économie sociale, de certaines règles qui génèrent des obstacles économico-financiers a permis d'améliorer la structure financière de ce type d'entreprises. C'est le cas de la nouvelle réglementation liée aux dettes des travailleurs des SLab et des coopératives qui peuvent bénéficier du concours du Fonds de Garantie Salariale (FOGASA).

Un autre dispositif permet aux travailleurs ayant droit à l'allocation de chômage de choisir de capitaliser cette allocation en un 
Tableau 4 - Efficacité de la mesure 'Capitalisation en un paiement unique de l'allocation de chômage' (CAPD)

\begin{tabular}{|c|c|c|c|c|}
\hline \multirow[b]{2}{*}{ Année } & \multicolumn{2}{|c|}{$\begin{array}{l}\text { Nombre de STrab totaux des CTA } \\
\text { et SLab fondées chaque année }\end{array}$} & \multicolumn{2}{|c|}{$\begin{array}{l}\text { \% de STrab des nouvelles CTA et } \\
\text { SLab bénéficiaires de la CAPD }\end{array}$} \\
\hline & STrab de CTA & STrab de SLab & STrab de CTA & STrab de SLab \\
\hline 1991 & 6.261 & 4.473 & $29,7 \%$ & $80,1 \%$ \\
\hline 1992 & 8.409 & 4.928 & $47,0 \%$ & $67,3 \%$ \\
\hline 1993 & 12.188 & 6.336 & $43,5 \%$ & $77,1 \%$ \\
\hline 1994 & 13.491 & 6.805 & $41,7 \%$ & $75,9 \%$ \\
\hline 1995 & 12.449 & 4.930 & $38,4 \%$ & $67,0 \%$ \\
\hline 1996 & 10.463 & 3.442 & $43,7 \%$ & $84,2 \%$ \\
\hline 1997 & 9.375 & 4.779 & $41,9 \%$ & $66,5 \%$ \\
\hline 1998 & 7.447 & 11.307 & $45,0 \%$ & $49,2 \%$ \\
\hline 1999 & 6.870 & 11.814 & $50,0 \%$ & $50,1 \%$ \\
\hline 2000 & 8.483 & 12.306 & $49,5 \%$ & $53,2 \%$ \\
\hline
\end{tabular}

Source: Elaboration propre sur base des données du Ministère du Travail et des Affaires Sociales. STrab=Travailleurs associés.

paiement unique s'ils décident de fonder une CTA ou une SLab. Concernant ce dernier dispositif, le Ministère du Travail, par le biais de l'Institut National pour l'Emploi, paie aussi les cotisations à la Sécurité Sociale de ces travailleurs pendant la période théorique de perception de l'allocation chômage. Cette mesure n'exclut pas d'autres mesures auxquelles les travailleurs ou leurs entreprises auraient éventuellement accès.

L'impact de cette disposition légale s'est avéré très positif (voir Tableau 4). Environ 10.000 travailleurs en ont bénéficié chaque année.

Certaines mesures qui ont porté leurs fruits dans d'autres pays n'existent pas en Espagne. Citons la promotion via des fonds par exemple (Loi italienne 51/1992) ou encore la mise en œuvre de fonds visant à réactiver les entreprises en crise par le biais de coopératives (Loi Marcora de 1985 - Italie).

Les mesures évoquées jusqu'ici sont conçues pour améliorer la position financière de l'ES en soutenant directement la structure. Ce sont des politiques d'offre, généralement fondées sur des mécanismes financiers et de régulation peu coûteux et à charge des budgets publics.

Une autre batterie de mesures, plus coûteuses, concerne les fonds alloués par les Administrations publiques à la promotion et au développement de l'ES. En Espagne, le programme principal est celui du 'Développement de l'économie sociale' (Programme budgétaire 322C) de la compétence du Ministère du Travail, pour le développement de 
coopératives et de SLab. C'est, par ailleurs, une manière d'exploiter certains fonds européens provenant du Fond Social Européen (FSE). La plus grande partie de ces budgets pubics (entre $60 \%$ et $75 \%$ selon l'année) est transférée aux Communautés Autonomes suivant des critères objectifs, parmi lesquels, le taux de chômage régional et le niveau passé d'exécution budgétaire. L’Andalousie, la Catalogne, la Région de Valence et Madrid sont les principaux destinataires de ces transferts. A ces fonds transférés, les gouvernements régionaux ajoutent des fonds propres, selon les priorités de leur propre politique. Dans ce type de politique financière, trois niveaux de gouvernement sont donc impliqués, national, autonome-régional et européen, qui contribuent chacun de façon inégale. L'importance des fonds consacrés au développement de l'ES est limitée, en comparaison à d'autres politiques menées dans d'autres secteurs. Cette situation est dénoncée souvent par le secteur.

Les aides financières provenant de ces budgets sont généralement conditionnées à la réalisation de certaines activités (intégration de nouveaux travailleurs associés, intercoopération, restructuration de coopératives, etc.). Par ailleurs, certaines lignes de financement constituent des mesures véritablement innovatrices. Citons le cas de l'aide à la participation des travailleurs dans les entreprises au Pays Basque.

Nous définissons au Tableau 5 l'effort budgétaire de soutien de l'ES (EB) comme le rapport qui existe entre les fonds propres des gouvernements régionaux destinés à l'ES $(\mathrm{FP})$ et d'autres variables. Ainsi, EB1=FP/BGccaa où BGccaa représente le budget global de ces gouvernements de Communautés Autonomes; EB2=FP/BG ES où BG ES représente les fonds totaux ( $\mathrm{FP}+$ fonds transférés) destinés au soutien de l'ES dans la région; et finalement EB3=FP/PO qui représente l'effort budgétaire régional par travailleur occupé dans la région.

A la lumière de ce Tableau, il est aisé de constater que la contribution des régions autonomes est la source principale de financement de l'ES (EB2) mais qu'elle est très inégale entre les différentes régions. Les CCAA qui consentent les plus gros efforts budgétaires sur leurs propres fonds sont la Galice, la Murcie, l'Estrémadure, la Castille-La Mancha et l'Andalousie (voir colonne EB2, Tableau 5).

Existe-t-il un rapport entre le niveau d'effort budgétaire et les variables politiques et économiques régionales?

Le Tableau 5 nous a permis d'identifier un rapport direct entre le niveau de développement économique d'une région, mesuré par son PIB relatif par tête aux prix de marché (100=moyenne européenne), et les 
Tableau 5 - Evolution de l'économie sociale marchande et effort budgétaire de soutien de l'ES en Espagne

\begin{tabular}{|c|c|c|c|c|c|c|c|c|}
\hline & \multicolumn{3}{|c|}{ Espace systémique de l'ES } & \multicolumn{2}{|c|}{ Variables politiques et économiques régionales } & \multicolumn{3}{|c|}{ Effort budgétaire de soutien de l'ES (2000) } \\
\hline & $\begin{array}{l}\text { Emploi dans } \\
\text { l'ES(EES) }\end{array}$ & $\begin{array}{l}\text { EES/PO } \\
\%\end{array}$ & $\begin{array}{l}\Delta \mathrm{EES} / \mathrm{PO} \\
\%\end{array}$ & $\begin{array}{l}\text { PIB par tête aux prix du } \\
\text { marché }\end{array}$ & $\begin{array}{l}\text { Parti au gouvernement } \\
(1997-2000)\end{array}$ & $\begin{array}{l}\text { EB1 } \\
(\text { en \%) }\end{array}$ & $\begin{array}{l}\text { EB2 } \\
(\text { en \%) }\end{array}$ & $\begin{array}{l}\text { EB3 (en Euros par } \\
\text { travailleur occupé) }\end{array}$ \\
\hline C.A.Galice & 12409 & 1,26 & 0,328 & 69,5 & $\mathrm{PP}$ & 0,29 & 92,9 & 18,4 \\
\hline C.A.Andalousie* & 59386 & 2,71 & 0,647 & 57,0 & PS & 0,17 & 88,2 & 12,5 \\
\hline C. de Valence & 48739 & 3,09 & 0,454 & 84,6 & PP-PRD & 0,14 & 85,6 & 6,4 \\
\hline C.A.Canaries & 10215 & 1,64 & 0,343 & 80,4 & PP-PRD & 0,09 & 80,3 & 5,4 \\
\hline C.A.Catalogne & 53236 & 2,13 & 0,207 & 103,6 & PRD (ClU) & 0,02 & 51,4 & 0,8 \\
\hline C.F.Navarre & 838 & 3,79 & 0,771 & 99,6 & PRD (UPN) & 0,07 & 86,1 & 6,7 \\
\hline C.A.Pays Basque* & 58116 & 7,24 & 1,651 & 92,6 & PRD (PNV) & 0,06 & 83,7 & 3,8 \\
\hline R.Murcie & 14547 & 3,49 & 1,278 & 67,4 & PP & 0,34 & 86,8 & 12,7 \\
\hline C.A.Extremadure* & 9119 & 2,65 & 0,501 & 56,6 & PS & 0,2 & 89,2 & 10,3 \\
\hline C.A.Castille-La Mancha & 20494 & 3,46 & 0,701 & 65,5 & PS & 0,18 & 83,7 & 8,1 \\
\hline P.Asturies & 5931 & 1,78 & 0,351 & 67,7 & $\mathrm{PP}$ & 0,13 & 76,5 & 4,9 \\
\hline C.A.La Rioja & 2344 & 2,39 & 0,441 & 97,5 & PP & 0,1 & 86,3 & 4,1 \\
\hline C.A.Aragon & 10874 & 2,37 & 0,355 & 90,6 & PP-PRD & 0,08 & 78,0 & 3,5 \\
\hline C.Madrid* & 19654 & 0,95 & 0,291 & 112,6 & $\mathrm{PP}$ & 0,04 & 61,1 & 1,1 \\
\hline C.A.I.Baléares & 2908 & 0,92 & 0,213 & 121,4 & PP & 0,02 & 62,1 & 0,7 \\
\hline C.A.Castille et Léon & 14993 & 1,74 & 0,246 & 74,8 & PP & 0,01 & 34,5 & 0,7 \\
\hline C.A.Cantabrie & 2001 & 1,1 & 0,192 & 75,2 & PP & 0,01 & 20,8 & 0,4 \\
\hline
\end{tabular}

Source: Elaboration propre sur base des données de l'Institut National de Statistiques, du Ministère du Travail et des Affaires Sociales, de la Direction Genérale de Développement de l'ES du FSE, www.map.es, estimation propre pour la Navarre et le Pays Basque, et Questionnaire CIRIEC.

Notes: (*) Pour l'homogénisation des données des régions, sur base de l'information du Questionnaire-CIRIEC, on a fait, pour l'Extremadure: réduction des montants destinés aux travailleurs indépendants; pour Madrid: exclusion des montants destinés aux Handicapes et aux travailleurs indépendants; pour l'Andalousie: la donnée correspond au Programme 67 C 'Développement coopératif et communautaire' avec exclusion des activités de Formation Professionnelle et Occupationnelle; pour la Galice: exclusion des transferts de l'Etat pour le soutien aux travailleurs indépendants. Pour la Navarre et Euskadi, les montants des fonds de soutien de l'ES transférés par l'Etat ont été estimés suivant une procédure à l'instar de celle qui a été appliquée dans les autres CCAA. 
trois indicateurs d'effort budgétaire. En effet, les régions les plus pauvres ont davantage tendance à soutenir l'ES. Ceci est cohérent avec leur façon d'appréhender la politique de développement de l'ES conçue comme un outil de création d'emploi et de tissu entrepreneurial.

Il est en revanche plus difficile à partir de nos données de vérifier l'hypothèse de Kirschen (1969) selon laquelle il existe des préférences idéologiques en faveur de certains objectifs de politique économique. Notre étude a permis de constater que les gouvernements conservateurs (au Tableau 5 PP représente le Parti Conservateur et PRD les Partis régionaux de droite) présentent un large éventail d'effort budgétaire: en effet, face à la Galice et la Murcie, qui présentent les plus grands efforts budgétaires du pays, d'autres (comme la Cantabrie et la Castille-Leon) sont les plus faibles. De l'autre côté, nous retrouvons les trois gouvernements régionaux socialistes (PS) parmi les 5 régions qui consentent l'effort budgétaire le plus important, mais ce sont également les régions dont le niveau de PIB par tête est le plus bas. Le cas des Iles Baléares - la région dont le niveau de PIB est le plus élevé - est particulier : la multiplication par trois, ces dernières années, de ses FP a été accompagnée d'une batterie de mesures en faveur de l'ES. Ceci s'explique par la nouvelle alliance de gauche qui s'est installée en 1999 au gouvernement.

Existe-t-il un rapport entre le niveau d'effort budgétaire consacré à l'ES et l'expansion de celle-ci?

L'étude révèle qu'il existe effectivement une relation positive entre les deux variables. Les trois régions qui ont consenti l'effort budgétaire le moins important présentent aussi les niveaux les plus bas de développement de l'ES et vice-versa. Citons l'exception du Pays Basque et de la Navarre où la dynamique du secteur de l'ES est puissante (grâce au Groupe MCC) et capable de s'auto-reproduire et de s'étendre. Le résultat est également lié à la nature des aides utilisées par les entreprises d'ES à savoir essentiellement les subventions pour la promotion de l'emploi, comme nous l'évoquons ciaprès.

L'impact sur les CTA et les SLab de ces lignes budgétaires de soutien a été mesuré récemment dans la Communauté Valencienne (Tomás-Carpi et Monzón, 1998). 57\% des CTA et 77\% des SLab actives en 1995 reconnaissaient avoir bénéficié de l'aide de l'administration publique, surtout au moment de la fondation de l'entreprise et pendant la première année d'activité. Les aides les plus demandées par les CTA sont les aides financières de promotion de l'emploi.

A côté de ces aides financières, soulignons encore l'importance de deux autres outils. D'abord, la régulation adoptée par l'Etat et les 
CCAA qui régit l'accès aux prêts et pose souvent des problèmes d'incompatibilité d'accès entre certaines lignes, incompatibilité manifeste dans certaines CCAA, (aux Canaries, en Galice et CastilleLeón par exemple) mais pas dans d'autres (Aragon 2000). Ensuite, le degré d'exécution des budgets qui prend une tournure préoccupante: en l'an 2000, 40\% environ des budgets du programme 322-c n'a pas été exécuté contre $23 \%$ en 1999. Enfin, signalons un dernier aspect : le retard des pouvoirs publics en matière de paiement.

\subsection{Politiques de soutien technique}

En Espagne, les pouvoirs publics concernés ont adopté un rôle proactif soutenant directement le secteur de l'ES en mobilisant des ressources matérielles et humaines dans le but d'offrir au secteur un éventail de services réels (non financiers).

Les différences entre administrations sont apparues dans les actions menées, tant au niveau de la nature institutionnelle de l'acteur qu'au niveau du type de services prestés. En effet, dans certains cas c'est l'initiative exclusivement publique qui a pris le dessus. Citons l'IPFC (Institut de Promotion et de Formation Coopérative), qui dépend de la Generalitat de Catalogne, les initiatives des Ecoles d'Entreprises du Gouvernement de l'Andalousie et les Centres d'Initiatives Entrepreneuriales d'ES de Castille La-Mancha. Dans la plupart des autres cas, l'implication du secteur de l'ES a été recherchée, de concert avec d'autres partenaires comme les Syndicats. Citons le cas de la Fondation pour le Développement de l'ES des Asturies.

En général, ces dispositifs deviennent opérationnels grâce aux structures représentatives du secteur (fédérations et confédérations) qui, en grande partie financées par les fonds publics, ont non seulement rempli la mission politique et de représentation qui leur est propre mais ont également assumé la fonction de prestation des services réels évoqués ci-dessus. La mise en œuvre de ce type de politiques est coûteuse en terme de budget, elle est donc limitée dans la pratique par la politique financière budgétaire présentée à la section précédente.

\subsection{Politiques de demande}

Exception faite du domaine des services sociaux, les politiques de demande en faveur de l'ES ne se sont que très peu développées en Espagne. En effet, on observe un nombre croissant de contrats passés 
entre les pouvoirs publics et les organisations de l'ES dans ce domaine et de subventions conditionnées à la réalisation de services sociaux. Mais l'institutionnalisation de ces pratiques est récente. Certaines mesures ont été adoptéés; c'est le cas, par exemple, de la préférence accordée aux coopératives en cas d'égalité dans le cadre de contrats des administrations publiques.

\section{Conclusion}

Cette étude s'est intéressée aux politiques économiques mises en œuvre dans le secteur de l'économie sociale déployées en Europe et plus spécifiquement en Espagne. Elle cherchait à examiner les différents types de politiques adoptées, à évaluer l'efficacité des principales mesures de soutien mises en place en Espagne et à déterminer le rapport entre l'effort budgétaire consacré au secteur de l'ES et certaines variables politique et économique.

Nous avons proposé, en premier lieu, un cadre d'analyse qui distingue trois typologies de politiques publiques. La première typologie distingue les politiques d'offre et les politiques de demande. Les politiques d'offre visent les structures des organisations de l'économie sociale tandis que les politiques de demande portent sur leurs activités économiques. La deuxième typologie différencie le caractère transversal du caractère sectoriel des politiques. Finalement, la troisième classe l'ensemble des politiques en fonction de leur orientation globale et distingue les politiques d'ordonnancement des politiques de processus.

Notre étude a mis en évidence que les politiques menées en Europe se déploient de façon inégale entre les pays et selon les branches de l'économie sociale. Deux modèles de développement de ces politiques ressortent. On observe d'une part, le modèle des pays méditerranéens latins (France, Italie, Espagne et Portugal) où ces politiques sont plus avancées présentant une institutionnalisation qui s'inscrit dans la Constitution Nationale et un biais favorable aux coopératives. D'autre part, on distingue le modèle des pays germano-britanniques qui se caractérise par un faible déploiement peu institutionnalisé des mesures d'appui, par un biais favorable aux organisations à but non lucratif et par un moindre soutien aux coopératives.

L'analyse plus approfondie des politiques menées vis à vis du secteur de l'économie sociale marchande en Espagne a permis de constater qu'il s'agit bien d'un pays qui répond au modèle méditerranéen latin. L'institutionnalisation des politiques y est 
avancée, notamment grâce à la reconnaissance légale de l'identité différenciée des entreprises du secteur et à leur fiscalité très favorable. Cependant, des carences se manifestent dans l'institutionnalisation du secteur comme interlocuteur de la concertation sociale dans le cadre de l'élaboration et de l'application des politiques, notamment celles de l'emploi.

L'obtention de ressources financières par les coopératives a été améliorée par les différentes politiques financières mises en place. Certains dispositifs, comme les subventions pour la promotion de l'emploi coopératif ou comme la possibilité de recevoir en un paiement unique l'allocation de chômage pour les travailleurs qui décident de fonder une coopérative de travail ou une société de travailleurs, ont fait preuve d'une grande efficacité. Par rapport à d'autres pays latins comme la France et l'Italie, des améliorations dans la définition d'autres dispositifs financiers et leur application devraient encore être introduites.

Les politiques de soutien technique au secteur se sont déployées dans toutes les régions d'Espagne, mais des différences existent dans leur application. Si certains dispositifs mobilisent en effet l'initiative exclusivement publique, la plupart des dispositifs tendent à impliquer les structures représentatives du secteur (fédérations, unions) pour offrir l'éventail de services. Enfin, exception faite du domaine des services sociaux, les politiques de demande en faveur de l'économie sociale ne se sont que très peu développées.

Par ailleurs, l'étude portant sur l'Espagne a permis de constater qu'il existe un rapport entre le niveau de développement économique d'une région, mesuré à partir du PIB par tête et le niveau d'effort budgétaire consacré à l'ES. En effet, les régions les plus pauvres tendent à soutenir financièrement l'économie sociale. Il semble exister aussi une relation positive entre l'effort budgétaire et l'expansion de l'emploi du secteur. Il ne nous a cependant pas été possible de vérifier l'hypothèse de Kirschen selon laquelle il existe des préférences idéologiques en faveur des politiques de soutien à l'économie sociale.

\section{RÉFÉRENCES}

AAVV, 2000, La contratación pública como instrumento de inserción sociolaboral, Instituto Navarro de Bienestar Social, http:// www.terra.es/personal3/redpobreza. 
ARAGÓN J., dir, 2000, Pactos y medidas de fomento del empleo en las Comunidades Autónomas, Ministerio de Trabajo y Asuntos Sociales, Madrid.

BAREA J. et MONZON J. L., dir, 2002, Summary of the Report on the Social Economy in Spain in the year 2000, CIRIEC-España ed, Valencia (Spanish and French versions available).

CHAVES R., 1999, 'Tejiendo redes entre pymes. La política y las entidades de promoción de la cooperación', in Prieto J. A., coord., Sociedades cooperativas: régimen jurídico y gestión económica, Ibidem et al., Madrid.

CHAVES R. et MONZÓN J. L., 2000, 'Politiques publiques', in CIRIECInternational, Les entreprises et organisations du Troisième Système (2 Vol.), CIRIEC-International, Bruxelles, Chap. 4.

CHAVES R., MONZON J. L., TOMAS-CARPI J. A., 1999, 'La Economía social y la política económica', en Jordán J. M. et al., (coord), Política económica y actividad empresarial, Tirant lo Blanch ed, Valencia, 143170.

CIRIEC-International, 2000, Les entreprises et organisations $d u$ Troisième Système (2 Vol.), CIRIEC-International, Bruxelles (Spanish version: Economía Social y empleo en la Unión Europea, Ciriec-España edl, Valencia).

CLOTFELTER C. T., 1985, Federal tax policy and charitable giving, University of Chicago Press, Chicago.

Comisión de las Comunidades Europeas, 1997, El fomento del papel de las asociaciones y fundaciones en Europa, Comunicación, COM (97) 241 Final.

COX H., 1997, 'Paradigm change in german economic policies', in Monnier L., Thiry B., ed., Annals of Public and Co-operative Economics, 68 (3), 355-366.

CUADRADO J. R., coord., 2001, Política económica, McGrawHill, Madrid.

Dirección General de Fomento de la Economía Social, 2001, Información sobre Economía Social ante los PNA para el empleo y para la inclusión social, fotocopiado.

DEFOURNY J., MONZÓN J. L., eds., 1992, Economie Sociale: Entre économie publique et économie capitaliste, De Boeck, Bruxelles.

DIES., Higher Council for Co-operation, 2001, Co-operative movements in the European Union, Ministère de l'emploi et la solidarité France, Paris. 
DOBKIN P., 1988, 'Private philanthropy and public policy: a historical appraisal'; in AAVV, Philanthropy, four views, Transaction books, London, 39-72.

FRAISSE L., GARDIN L. et LAVILLE J. L., 2001, 'Les externalités positives dans l'aide à domicile: une approche européenne', in LAVILLE J. L., NYSSENS M., dir., Les services sociaux entre associations, Etat et marché, La Découverte - Mauss - Crida, Paris, 192-207.

FREGIDOU-MALAMA M., 2000, 'The relationship between agricultural cooperatives and the State in Sweden. The legislative process', Annals of Public and Co-operative Economics, 71 (1), 79-104.

JAMES E. et ROSE-ACKERMAN S., 1986, The nonprofit enterprise in market economies, Harwood Academic Publishers, Switzerland.

JANE SOLA J., 1979, 'Por una estructura operativa de la política económica', in Irastorza J., coord., Política económica, CECA, Madrid.

JORDAN J. M. et al., coord, Política económica y actividad empresarial, Tirant lo Blanch, Valencia.

JULIA J. F. et SERVER R., 1996,'La fiscalidad de las cooperativas en el marco de la Unión Europea. Referencia especial al caso español', Ciriec-España, 23, 47-70.

KENDALL J. et ANHEIER H. K., 1999, 'The third sector and the European Union policy process: an initial evaluation', Journal of European Public Policy, 6 (2), June, 283-307.

KNAPP M., ROBERTSON E. et THOMASON C., 1990, 'Public money, voluntary action: whose welfare?', in Anheier H., Seibel W., ed., The Third Sector: comparative studies of nonprofit organizations, W. De Gruyter, Berlin.

LUNARIA., Universidad de Roma., 1998, The Third Sector in Europe: review of present policies, Lunaria, Roma.

MONNIER L. et THIRY B., eds, 1997, 'Structural changes and general interest: which paradigms for the public, social and cooperative economy?', Annals of Public and Co-operative Economics, 68 (3), september.

MONZON J. L., 1997,'Contributions of the social economy to the general interest', Annals of Public and Co-operative Economics, 68 (3), 397408.

OIT., 2001, Informe V. Promoción de las cooperativas, Oficina Internacional del Trabajo, Ginebra (2 vol.).

Revue NOTICIAS de la economía pública, social y cooperativa, plusieurs números, CIRIEC - España, Valencia. 
ROJO E. et VIDAL I., 1988, Medidas de apoyo a las empresas de trabajo asociado, Ministerio de Trabajo y Seguridad Social, Madrid.

ROSE-ACKERMAN S., 1986, ed., The economics of nonprofit institutions. Studies in structure and policy, Oxford University Press, Oxford.

RUIZ OLABUÉNAGA J. I., dir, 2000, El sector no lucrativo en España, Fundación BBV, Bilbao.

SAJARDO A., 1996a, Análisis económico del sector no lucrativo, Tirant lo blanc, Valencia.

SAJARDO A., 1996b, 'Una panorámica del tratamiento fiscal a la Economía Social en España', Ciriec-España, 23, 11-32.

SIMON J. G., 1987, 'The tax treatment of nonprofit organisations: a review of federal and states policies', in Powell W., ed., The nonprofit sector: a research handbook, Yale University Press, New Haven, 67-98.

SIMON J. G., 1990, 'Modern welfare state policy toward the nonprofit sector: some efficiency-equity dilemmas', in Anheier H., Seibel W., ed., The Third Sector: comparative studies of nonprofit organizations, W. De Gruyter, Berlin, 31-52.

THIRY B. et VIVET D., 2000, 'Champ de l'étude, importance quantitative et acceptions nationales', in CIRIEC-International, Les entreprises et organisations du Troisième Système (2 Vol.), CIRIECInternational, Bruxelles, Chap. 1.

TOMAS-CARPI J. A., 1990, Política económica: su lógica, estructuración $y$ aspectos metodológicos, Dpto Economía Aplicada - Política Económica, Universitat de Valencia, Valencia.

TOMAS-CARPI J. A., 1997, 'The prospects for a social economy in a changing world', Annals of Public and Cooperative Economics, 68 (2), 247-279.

TOMAS-CARPI J. A., MONZÓN J. L., dir., 1998, Libro blanco de la Economía Social en la Comunidad Valenciana, Ciriec-España ed., Valencia.

VANBERG V., 1988, 'Ordnungstheorie as constitutional economics. The german conception of "socialmarket economy", Ordo, Band 39, 17-31.

VIENNEY C., 1985, 'L'organisation coopérative comme instrument de politique économique', RECMA Revue internationale de l'économie sociale, $16,55-64$. 


\section{Public policies and social economy in Europe. The Spanish case}

The article considers different economic policies likely to affect the social economy sector as an institutional sector. In a first time it sets up an analytical framework which allows to apprehend the policies contents and offers three typologies. In the light of this framework it first makes a global evaluation of policies implemented in Europe and then focuses on Spain, one of the Union countries with the highest level of development of these policies. Finally, the author evaluates the efficiency of the main measures of economic policy applied in this country.

\section{Staatliche Politik gegenüber der Sozialwirtschaft in Europa. Der Fall Spanien}

In diesem Beitrag werden verschiedene Wirtschaftspolitiken betrachtet, von denen anzunehmen ist, dass sie den sozialwirtschaftlichen Sektor als institutionellen Sektor beeinflussen. Eingangs wird ein analytischer Rahmen geschaffen, der es ermöglicht, die Politikinhalte festzustellen, und es werden drei Typologien angeboten. Innerhalb dieses Rahmens wird zuerst eine globale Evaluation von Politiken vorgenommen, die in Europa praktiziert werden, und es wird dann der Fokus auf Spanien gerichtet-eines der Länder der Union mit dem höchsten Entwicklungsstand hinsichtlich dieser Politiken. Schließlich bewertet der Autor die Effizienz der wichtigsten Maßnahmen der ökonomischen Politik, die in diesem Land Anwendung findet.

\section{Politicas publicas y economia social en Europa. El caso español}

Este artículo examina las diferentes políticas económicas susceptibles de incidir sobre la economía social como sector institucional. En primer lugar se ofrece un marco analítico que permita aprehender los diferentes contenidos de estas políticas asi como edificar una tipología de las mismas. A la luz del marco anterior, se realiza un balance global de las políticas implementadas en la Unión Europea. El análisis se efectúa posteriormente con mayor detalle para el caso de España, uno de los países de la Unión con un nivel más avanzado de desarrollo de estas políticas. Se valorará finalmente la eficacia de las principales medida de politica económica puestas en marcha en este país. 